\title{
Barriers to Predicting the Mechanisms and Risk Factors of Non-Contact Anterior Cruciate Ligament Injury
}

\author{
Nicholas Ali*,1 and Gholamreza Rouhi ${ }^{2,3}$ \\ ${ }^{1}$ School of Human Kinetics, University of Ottawa, Ottawa, Canada \\ ${ }^{2}$ Department of Mechanical Engineering, University of Ottawa, Ottawa, Canada \\ ${ }^{3}$ Faculty of Biomedical Engineering, Amirkabir University of Technology, Iran
}

\begin{abstract}
High incidences of non-contact anterior cruciate ligament (ACL) injury, frequent requirements for ACL reconstruction, and limited understanding of ACL mechanics have engendered considerable interest in quantifying the ACL loading mechanisms. Although some progress has been made to better understand non-contact ACL injuries, information on how and why non-contact ACL injuries occur is still largely unavailable. In other words, research is yet to yield consensus on injury mechanisms and risk factors. Biomechanics, video analysis, and related study approaches have elucidated to some extent how ACL injuries occur. However, these approaches are limited because they provide estimates, rather than precise measurements of knee - and more specifically ACL - kinematics at the time of injury. These study approaches are also limited in their inability to simultaneously capture many of the contributing factors to injury.

This paper aims at elucidating and summarizing the key challenges that confound our understanding in predicting the mechanisms and subsequently identifying risk factors of non-contact ACL injury. This work also appraise the methodological rigor of existing study approaches, review testing protocols employed in published studies, as well as presents a possible coupled approach to better understand injury mechanisms and risk factors of non-contact ACL injury. Three comprehensive electronic databases and hand search of journal papers, covering numerous full text published English articles were utilized to find studies on the association between ACL and injury mechanisms, ACL and risk factors, as well as, ACL and investigative approaches. This review unveils that new research modalities and/or coupled research methods are required to better understand how and why the ACL gets injured. Only by achieving a better understanding of ACL loading mechanisms and the associated contributing factors, one will be able to develop robust prevention strategies and exercise regimens to mitigate non-contact ACL injuries.
\end{abstract}

Keywords: Anterior cruciate ligament, non-contact ACL injury, injury mechanisms, risk factors.

\section{BACKGROUND}

A non-contact injury is defined as an injury that occurs without physical contact with another person or object [1]. Studies have demonstrated that 70 to $80 \%$ of ACL injuries occur during non-contact sporting events, which involve sudden deceleration, an abrupt change in direction, or jump landing as is common in soccer, basketball, handball, and volleyball [2-4]. Increased participation in athletic activities, especially among females, and the growing number of people in sports has resulted in an increase in the number of ACL injuries. Among athletes, females are 2-8 times more likely to sustain a non-contact ACL injury than males [5-8]. Approximately 100,000 to 175,000 ACL-related surgeries are conducted in the United States each year [9], with associated costs exceeding $\$ 2$ billion. Complete ACL rupture may lead to significant posttraumatic laxity, functional knee instability, and increased likelihood of osteoarthritis [10-13]. Advances in surgical techniques and rehabilitation have resulted in ACL surgery becoming a relatively routine procedure [2].

*Address correspondence to this author at the School of Human Kinetics, University of Ottawa, Ottawa, Canada; Tel: 1-613-562-5800 ext. 4246;

Fax: 1-613-562-5149; E-mail: nali065@uottawa.ca
During the 1990s, there was a paradigm shift in research focus on the ACL, from the development of more effective diagnostics and treatments to greater emphasis on identifying the mechanisms and risk factors of non-contact ACL injuries [14]. This shift was driven by the realization that only if the causal relations between applied forces and the resultant injury are understood, then appropriate programs of intervention and prevention can be designed and implemented [15]. This focus persists, but progress in this research area has been somehow patchy and somewhat slow. Nonetheless, the prevention of sport related non-contact ACL injuries today relies largely on the ability to screen at risk individuals and then modify through training the identified risk factor. Neuromuscular control strategies and muscle strength, or more specifically, proprioceptive training and hamstring strength training respectively, are among the risk factors that can be modified through training [16-18].

The goal of this work is to provide a comprehensive assessment and summary of the challenges that hinder our ability to precisely determine injury mechanisms and risk factors of non-contact ACL injury. This is a crucial task since, as an unfortunate fact, the literature lacks consensus and coherence with regards to predicting injury mechanisms and determining risk factors of non-contact ACL injuries. 
Understanding these challenges is also a necessary first step towards designing studies to better understand non-contact ACL injury mechanisms and the associated risk factors, as well as, subsequently enabling the development of injury prevention programs. This article also highlights the grave needs and promising opportunities to steer future research direction in non-contact ACL injury biomechanics. It focuses on one important question, that is, what is the current status in clearly predicting the mechanisms and risk factors of noncontact ACL injury?

\section{MATERIALS AND METHODS}

This article reviewed the relevant literature on ACL injury mechanisms in the PubMed electronic database using MEDLINE (1966 to 2010), Proquest (1987 to 2010), and, Applied and Complementary Medicine Database (AMED) on Ovid (1985 to Sept. 2010). Keywords used in our search included "anterior cruciate ligament", "ACL injuries", "injury mechanisms", "risk factors", and "non-contact injuries". A total of 813 articles were identified and reviewed. The most relevant full text English articles pertaining to ACL non-contact injuries, risk factors for ACL injuries, and study approaches to understand ACL mechanics were analyzed. Studies that captured the association of ACL and non-contact injuries, and ACL with a specific study approach were also included. Our search was supplemented by reviewing the bibliographies of retrieved articles, as well as, hand searching scholarly journals outside of the bio-fields related to this topic.

\section{CHALLENGES IN IDENTIFYING MECHANISMS AND RISK FACTORS OF NON-CONTACT ACL INJURY}

The mechanism of injury to the ACL is rather complex because the injury event is quick, the injury condition is dynamic, and the knee is a complex joint capable of interrelated compression, translation and rotation about various axes [18, 19]. The mechanism of ACL injury is also complex because it involves the concurrent interaction of muscle forces, external forces, ligament forces, and joint contact forces. Several studies [2, 4, 20-26] have suggested several non-contact ACL injury mechanisms: jumping, landing, side step cutting maneuvers to name a few. Most study approaches have provided valuable information but do not offer a comprehensive view of ACL injury mechanism. The literature also reports a host of risk factors contributing to ACL injuries and as such demands the use of a method that accounts for all of these factors at the same time, and not only examine the biomechanics associated with injury or individual or external risk factors in isolation [27]. The following review articles [7, 8, 28-30] has compiled and summarized the risk factors to non contact ACL injury. So even though a recent thorough review [27] has shown that ACL injuries occur from a complex interaction of multiple risk factors, very few studies in the literature take this into account.

Fragmentation and discrepancies in the literature may be a reflection of the limitations and differences in current noncontact ACL injury study approaches which includes but not limited to, equipment used, computing power and software programs. More importantly, little is known about ligament and muscle loading and response during ACL injuries. This may be attributed to the innate difficulties associated with measuring ligament and muscle forces in-vivo. It is understood, based on this review, that most of the research on non-contact ACL injuries has focused on the effects of knee mechanics before and after ACL rupture, or before and after ACL reconstruction, but not on the very important aspect of precisely how and why the ACL gets injured.

Consequently, the success of any screening method relies on a precise understanding of the relationship between knee kinematics, joint geometry, external loading, neuromuscular control, ACL loading, and the associated contributing risk factors of injury. Until accurate descriptions of these relationships are available, the potential exists for all screening methods to exclude incorrectly "at risk" people from any ensuing intervention process [31, 32]. For athletes who are at higher risk, a complete understanding of injury causation needs to address the multi-factorial nature of sport injuries $[33,34]$. Even though a multidisciplinary approach is recognized as a more robust study approach by some research groups [35-37] it has never been undertaken. The following section highlights some of the major impediments to furthering our understanding of non-contact ACL injury mechanisms and risk factors.

\subsection{Shortcomings and General Inherent Challenges of Studying Non-Contact ACL Injury}

For ethical reasons, in-vivo measurements of ACL loading to failure in human or animal subjects cannot be undertaken. Hence, relationships between internal forces, external loading, and ACL loading mechanism are mainly unknown due to the difficulties of measuring ligament and muscle forces in-vivo. Moreover, maximum kinematic changes of knee, for example, anterior tibial translation (ATT) may not necessarily correspond to maximum force in the ACL due to the concurrent interactions of multiple tissues surrounding the knee. These interactions are very difficult to capture even with the most sophisticated existing study approach. The problem is complicated further by the structural complexity of the knee and the ACL, as well as, the multiple structures that contribute to similar function. The difficulties in obtaining material property data for the hard and soft human tissues also create some limitations, especially for computational modeling studies. Other challenges include high cost of experimental studies, as well as, the difficult and sometimes impossible task of reproducing certain natural, pathological or degenerative situations in-vitro. In addition, there can be significant interand intra- subject variability during experimental testing. The following subsections elucidate specific areas where challenges and difficulties in studying non-contact ACL injury persist.

\subsubsection{Study Approach do not Capture Muscle Activation and Kinematics}

Study approaches that do not include muscles may not adequately predict injury mechanisms and risk factors of non-contact ACL injury. Omitting muscles leads to inaccuracies since the forces transmitted to the ligaments and bones are dominated by the muscle forces [38]. It appears that ligaments can only play a limited role in maintaining the integrity and stability of the knee joint. In fact, it is likely that ligament rupture occurs only when muscle action fails to 
protect them. Muscle recruitment strategies may also be one indicator why females suffer more non-contact ACL injuries than men [39-42], and strongly points to the importance of musculo-tendon contributions in maintaining knee joint stability and integrity. Hence, if for any reason the firing pattern and the magnitude of the load by which the muscle pulls on the bones of the knee are improper, then the ACL will then have to take the brunt of the load and in some instances will rupture.

In-vivo studies [43] have concluded that ACL strain increases if there is an increase in quadriceps activity relative to hamstring activity. McConkey [44] are the first to describe eccentric quadriceps contraction as the intrinsic force responsible for ACL injury. Numerous in-vitro studies using defect free cadavers have also focused on the effects of increased quadriceps forces on ACL strain [45, 46] and concluded that high unopposed quadriceps forces induce ATT, and consequently increase the strain in the ACL over a specific range of knee flexion angles. One in-vivo study with healthy men and women (using 11 subjects) aged 21-42 years and with normal ACL [47] showed similar results. However, one in-vitro study using six pairs of human cadaveric knees [48] found that the quadriceps muscle force protected the ACL from injury. As well, one study demonstrated that under non-physiological loading without any ground reaction forces (GRFs), the knee locked in one position, and static quadriceps loads of $4500 \mathrm{~N}$ applied, ACL injury did occur [49]. The authors suggested quadriceps drawer as an injury mechanism. Hamstring muscle force applies a posterior directed force component and has been reported to strain shield the ACL by reducing ATT at large flexion angles [50]. An early study by Solomonow et al. [51] suggests that strength training of the hamstring muscles can help prevent damage to the intact ACL. On the other hand, a later study by Shelburne [52] demonstrated that the hamstring cannot apply large enough posterior forces to unload the ACL especially at low knee flexion angles- a position where many non-contact ACL injuries occurs. It has also been demonstrated by Simonsen et al. [53] that the ability of the hamstring muscles to reduce the ACL load is marginal.

The results of an in-vivo study by Fleming et al. [54] demonstrated that the gastrocnemius muscle is an antagonist to the ACL. This is in agreement with an analytical study by Pflum et al. [55]. On the other hand, a cadaveric study by Durselen et al. [56] and a theoretical investigation by Shelburne [57], both demonstrated that the contraction of the gastrocnemius muscles did not strain the ACL over the entire range of knee flexion.

The discrepancies among these studies highlight the need to determine the contribution of muscle activation and loading on ACL loading. Nonetheless, many studies clearly demonstrate that ACL loading mechanism is affected by muscle activity and that the muscles must be included in these investigations. Moreover, considering the limited strength of the ACL, active control of the knee joint depends on the balance of the resultant muscle forces.

\subsubsection{Lack of Studies that Include Ankle and Hip Articulation}

The majority of non-contact ACL injury studies do not address the effects of hip and ankle kinematics and kinetics on injury. In addition, the majority of studies in the literature do not account for the effects of whole body movement on ACL loading. The inclusion of the ankle is important in noncontact ACL injury studies as it has been shown that the interaction of shoe surface interface with the ground is an important risk factor in non-contact ACL injury [24]. An increase in the coefficient of friction between the shoe and playing surface may increase the traction which can cause the foot to catch or stop inadvertently during play. Such types of events are associated with higher risk of ACL injury. By one study conducted using soccer players with varying cleat lengths, it was also shown that foot contact with the ground is an important risk factor in non-contact ACL injury [58]. Lambson et al. [58] also demonstrated that shoes with more cleats results in higher torsional resistance at the foot-surface interface which can result in increased risk of ACL injury. As well, muscle activity across the ankle controls the position of the foot at landing, which most likely influence the loading at the ankle [59]. Moreover, muscular activity at the ankle may influence the loads that are transferred through the ankle to the knee [59, 60]. Pflum et al. [55] found that the forces applied by the ground to the foot have a major impact on peak forces seen at the ACL. Another study found that when the foot was not flat, the ground reaction forces (GRFs) cannot be transmitted effectively through the bones to the ground without the actions of muscles [61]. In addition, excessive foot pronation was shown to increase the likelihood of non-contact ACL injury [62].

Inclusion of the hip articulation is also important to the understanding of how and why the ACL gets injured since hip movement is known to affect the loading on the knee [40]. Because the upper body contains over half of the total body mass, the trunk and pelvis position will have coupled effects on knee angles and resultant ACL strain. The position of the leg at the time of non-contact ACL injury displays tibial rotation, apparent knee valgus, foot pronation, and a relatively extended knee and hip [4, 14]. Muscle contraction at the hip can also affect knee loading because the hip transfers the upper body loads to the leg [6]. In addition, muscle contraction at the hip can also affect energy absorption [63, 64]. Moreover, a cadaveric study [19] and an analytical study [65] both demonstrated that if the hip movement is restricted, ATT will result and ACL injury can easily occur. Some other studies also suggested that the knee is one part of the kinetic chain and that the torso, hip, and ankle may also contribute to ACL injury [40, 66]. The importance of including the hip and ankle joints in any noncontact ACL injury study is supported by many other studies which are captured in a review article by Hewett et al. [30]. So, one can conclude that non-contact ACL injury is a whole body event that requires the study approach to capture the contributing effects of ankle and hip articulations on ACL loading.

\subsubsection{Studies do not Accurately Capture Knee Joint Geometry}

Specific contours of the knee joint geometry, for example, the femoral notch [67] has been implicated as a risk factor for non-contact ACL injury. The complex geometry of the knee joint aids in stability. The femoral groove that the patella slide on, the femoral condyles resting on the tibial 
eminence [68] and the posteriorly sloped tibial plateau [69] are all factors that may affect loading seen at the ACL during various non-contact ACL injury mechanisms. There are many computational studies that overlook the contribution of these factors to ACL injury. In fact, Liu [70] found that articular surface geometry, ligament locations, and ligament insertion sites had a pronounced effect on the modeling outputs. Irrespective of muscular activity, Rentrom et al. [71] demonstrated that the ACL is subjected to an inherent increase in strain as the knee extends owing to the geometry of the articular surfaces of the knee. Despite this, many studies have not considered accurate three dimensional (3D) hard and soft tissue geometries and few have investigated the effect of geometry changes on loads seen at the ACL [7274]. The inclusion of accurate 3D tissue geometries appears to be a crucial non-contact ACL injury study approach prerequisite. Without an objective view of these parameters in problem definition, a clear and comprehensive understanding of injury mechanisms and risk factors of noncontact ACL injury may remain elusive.

\subsection{Limitations in Current Non-Contact ACL Injury Study Approaches}

Experiment, athlete interviews, clinical studies, video analysis, and computational modeling are all different study approaches that have contributed to the understanding of ACL mechanics (see Fig. 1). A concise overview of many of these approaches along with their strengths and weaknesses are presented by Krosshaug et al. [75]. It seems that one common shortcoming in all these approaches is the inability to fully capture the many factors implicated as contributors to non-contact ACL injury. Clinical studies, athlete interviews, and video analysis has provided mostly qualitative data and so are not adequate for obtaining a comprehensive understanding of injury to the ACL. From the current literature, one can glean two main quantitative study approaches for better understanding how and why the ACL gets injured, namely, experimentation and computational modeling (highlighted in green in Fig. 1). The existing quantitative study approaches shown in Fig. (1) will be discussed in the context of this review.

\subsubsection{Challenges Facing the Field of In-Vivo Experimentation}

It can be argued that historically research on the human musculoskeletal system was focused on empirical methods. One form of in-vivo testing entails the direct implantation of a transducer to subjects' tissues. These are termed contact methods and have the advantage of simultaneously including many risk factors, forces, the effects of active muscles, as well as weight-bearing loads. A significant challenge with this form of in-vivo testing is that it is invasive. Ethical policies controlling testing with humans make in-vivo testing extremely difficult. Moreover, because of the size of the

\section{EXISTING STUDY APPROACHES}

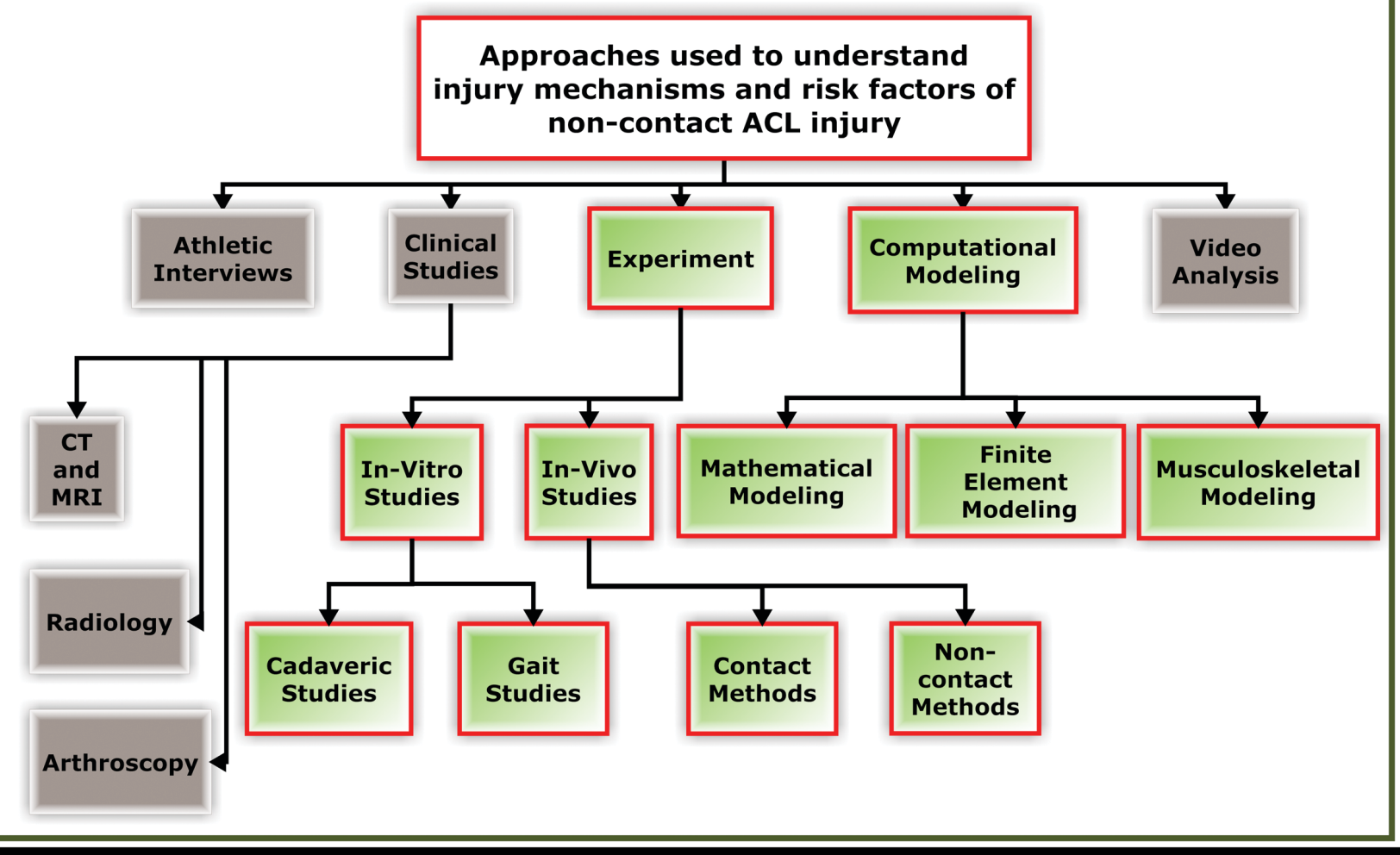

Fig. (1). Existing approaches employed to better understand mechanisms and risk factors of non-contact ACL injury. 
sensor employed, size of the ACL, intraarticular location of the ACL and intercondylar notch, in-vivo studies to-date can only measure strain on the anteromedial bundle of the ACL for movements confined to the sagittal plane [76]. Measurements are restricted to linear displacement at discrete locations, knee flexion angles approximately greater than 15 degrees, and movements only in the sagittal plane [47]. In addition, many of these studies look at movements that are non-ballistic. Woo et al. [77] have also argued that since strain gauges and other similar type transducers are implanted into the ligament, they can alter the ligament length, and subsequently ligament force. Another complicating factor with in-vivo techniques is the fact that specific bands of the ligament are tensioned at different portions of the loading cycle. Another form of in-vivo testing includes non-contact methods. These methods do not require the implantation of a sensor into the subject's ACL. Noncontact in-vivo methods include, but are not limited to, the use of dynamic magnetic resonance imaging (MRI) [78], biplane fluoroscopy [50,79-82] and video dimension analyzer [83] to capture tissue kinematics at various knee flexion angles. These techniques do not provide any details of the forces seen at the ACL during movement. Another, non contact method include optical techniques such as Roentgen Stereophotogrammetry $[84,85]$. Various contact techniques have been used in the past to measure in-vivo ACL strain: implantable extensometers [47, 71, 86-91] and buckle transducers [92-97]. It may be argued that none of these techniques are truly in-vivo given they alter in one form or the other the way the subject moves during testing.

\subsubsection{Challenges Facing the Field of In-Vitro Experimentation}

In-vitro testing is conducted outside of the body typically with human subjects or post mortem human subjects (PMHS)/ cadavers. The vast majority of studies investigating non-contact ACL injuries are in-vitro [19, 77, 98-100]. The major advantage of in-vitro testing is its utilization of biological tissue for testing. In-vitro testing also has the capability to simulate knee kinematics and muscle loads. However, in-vitro studies using cadavers may not accurately describe ligament function in-vivo since loading applied to the cadavers during experiment is different from that applied by the muscles during activity. Other challenges with invitro studies using cadavers are the inability to simulate realistic muscle activation and the difficulty in obtaining repeatable results. Furthermore, replicating an isolated ACL injury in-vitro is difficult, and has been achieved with only limited success [19]. This shortcoming may exist because cadaveric knee studies lack the stability and control provided by active knee musculature [101]. It is also difficult to apply large muscle forces seen during normal physical activities such as landing from a jump to in-vitro experiments using cadavers. This is partly due to the fact that many cadavers are from the elderly population. Another prevalent in-vitro study method is gait analysis that employs skin markers. The use of skin markers with gait studies have been shown to induce significant errors in predicting in-vivo ligament behavior [102-104]. Since some gait studies are conducted in the laboratory setting, there is also some level of uncertainty with respect to the ability of these tools in capturing true human response. Given the subject is aware that the camera is focused on them or their feet have to strike a force plate, it can be argued that their body response may be somewhat altered to fit the event. As a result, the data collected may not be a true representation of human motion. Despite its shortcomings, in-vitro studies have the capability to provide much freedom to investigate function and behavior of the ACL. From this standpoint, it can be argued that gait analysis is the only way available today to determine the kinematics and kinetics during activity to cause non-contact ACL injury.

\subsubsection{Challenges Facing the Field of Computational Modeling}

There are mainly three approaches to computational modeling in the current literature: mathematical, finite element (FE), and musculoskeletal rigid body (RB) modeling. A computational model of the knee joint is a graphical representation of the joint anatomy that can be manipulated. Computational modeling have become popular partly because technological restrictions and ethical considerations have prevented the direct measurement of the ACL strain in-vivo. Many mathematical models of the human knee focus on the intact ACL. With many of these models, if injury is studied, it is done primarily by removing the effect of the ACL in the model predictions [105]. Even though mathematical models have assisted us in gaining a better understanding of the mechanics of the knee, the gross approximations and assumptions made by these models can readily be addressed with technology and information available today. Two such technologies are FE and RB modeling. Mathematical models have become less popular over recent years perhaps due to: (1) Easy access and lower cost of FE and RB modeling software; and (2) Inherent complexity and nonlinearity in the governing equations related to ACL biomechanics, and subsequently the challenges entailed in developing and verifying these models. Hefzy [106] provided an excellent review of mathematical models of the human knee joint found in the scholarly literature

The first application of finite element analysis (FEA) to biomechanics was in orthopedics [107]. The most extensive application of FEA to knee biomechanics has been in artificial joint design and fixation. Two studies [108, 109] provided a brief review of the application of FEA to biomechanics. Andriachi et al. [110] was the first to develop a knee joint model for the determination of the forces using FE methods. Once verified and validated, a FE model can provide greater capabilities to answer many what if questions over mathematical models. Finite element models also have the advantage of being able to calculate parameters that are difficult to measure experimentally. The literature indicates that only a small number of FE models are used to study ACL mechanics, and none has focused on predicting mechanisms and risk factors to non-contact ACL injury (see for instance [72, 73, 111-128]).

Many of these FE studies do not report data on model verification and validation, thus limiting their use in clinical applications. Other FE studies investigating ligament mechanics in the literature modeled only the ACL [112, 129, 130]. None of these studies investigated non-contact ACL injury. Nonetheless, the challenge with FE modeling of biological tissues include the complexity of modeling 
material responses under loading, the level of discretization required to capture complex and intricate anatomical geometries, long computational time to converge [131], and dependence on empirical data for validation among others. Increased and affordable computing capacity and more sophisticated (nonlinear and 3D) FE software packages has allowed more realistic modeling and the application of iterative procedures to describe time-dependent biomechanical behavior. Nonetheless, this may not be enough to fully address the challenges posed by the complexity of predicting non-contact ACL injury mechanisms and risk factors.

Musculoskeletal RB modeling of the knee is based on research aimed at developing a dynamic rigid body model for balancing internal forces with externally applied forces to produce motion [132-134]. Three main approaches may be used to balance internal forces with externally applied forces for a specific motor task: inverse dynamics; forward dynamics; and optimum control theory. Erdemir et al. [88] provided a thorough review of these three approaches. The main advantage of musculoskeletal RB modeling is that it enables us to determine the forces in the muscles during activities implicated to cause non-contact ACL injuries. However, musculoskeletal models cannot provide details of the loads and stresses in the hard and soft tissues, like FE models can. In addition, RB musculoskeletal models can require extremely long computational time to converge to a solution and in some cases requires parallel computing [135].

\subsection{Shortcomings in Biomechanics Field}

The literature points to some systemic challenges facing the field of biomechanics which are believed to be impeding progress and clouding a collective agreement in the understanding of mechanisms and risk factors of non-contact ACL injury. ACL biomechanics research is mostly empirical. An empirical study approach has been the main method of building the foundation of biomechanics. In fact, there is a significant gap between the advancement of empirical tools and that of analytical and numerical tools in biomechanics. It has also been recognized that there is a lack of consensus in the research community on the merit of formulating clinical recommendations based on physical and numerical model results [136]. This may exacerbate itself to much uncertainty in theories used for teaching clinical biomechanics [137]. ACL injury research is a multidisciplinary field, since one need to consult with many disciplines in a single problem. With the cross linking of certain disciplines (such as biomechanical engineering, mechano-biology, bio-infomatics, etc.), problems in noncontact ACL injury research may become more easily solvable. Such cross linking may also bring about new resources, skill sets, approaches, and outlooks to the field of biomechanics.

The following two subsections shed some light on a few shortcomings in the field of biomechanics that continues to hinder a clear understanding of the mechanisms and risk factors of non-contact ACL injury.

\subsubsection{Studies Too Narrowly Focused}

Based on this investigation, it is understood that the type and complexity of the research method employed depends on the question(s) posed. Many studies have pointed out that several intrinsic and extrinsic factors are responsible for noncontact ACL injuries [30, 138, 139]. Other studies have categorized risk factors into four areas: environmental, biomechanical, neuromuscular, and hormonal [40]. However, many studies focused on investigating the effects of a single risk factor by comparing intrinsic or extrinsic differences between males and females, such as Q-angle [140], intercondylar notch width [141], and hormones [142] without considering the combined effects of these and other factors implicated to cause injury. It seems that the multitude of intrinsic and extrinsic risk factors involved during sports make focusing on one factor difficult. In addition, some studies address the effects of only one muscle group on ACL rupture. It seems quite unlikely that a single risk factor or only one muscle group will be responsible for ACL injury. There are few studies, to the best of our knowledge, where a tool or methodology is employed that lends itself to simultaneously determining the effects of numerous parameters on injury mechanisms and risk factors of noncontact ACL injury. Finally, the low annual incident rate for ACL injury in the general population of 1 per 3000 people [143] -and even smaller in the athletic population- has compelled researchers aiming at pinpointing factors contributing to risk of ACL injury to adapt a reductionist approach by focusing on a single factor or few factors due to small sample size. Given this, studies to date are narrowly focused due to statistical power limitations.

\subsubsection{Lack of Standardization}

Combining and comparing results from separate studies that use similar research methods can be valuable, but differences in, for example, specimen type and data reporting may prevent drawing solid conclusions about the results. The challenge is these differences may exist due to the lack of guidelines on study method, instrumentation to employ, and procedures for data analysis. The dearth of standards and specifications in the field of biomechanics is believed to be one reason why dialogue among research groups and comparisons in experimental studies remains challenging, debatable, and sometimes with no solid outcome. A few attempts at standardization that have led to considerable benefits in the research community include the Visible Human Project [144], VAKHUM [145], and the standardized femur [146]. These endeavors have aided in simplifying cross validation of research results. Due to the lack of standards and specifications in study approaches, accumulating knowledge on injury mechanisms and risk factors of non-contact ACL injury is believed to be slowed. As well, many of these studies are too highly focused and are not powered to handle the contribution of multiple factors on non-contact ACL injury.

\section{TOWARDS A BETTER UNDERSTANDING OF RISK FACTORS TO NON-CONTACT ACL INJURY}

Non-contact ACL injury research requires the use of many modalities, data from disparate sources, and many specialized software tools [37]. A possible approach is a coupled study approach that leverages on the advantages of existing non-contact ACL injury study approaches. For a list of existing study approaches used in non-contact ACL injury research see Fig. (1). Fig. (2) highlights the coupled study 
approach proposed. This approach entails utilizing a 3D electromyography (EMG)-driven FE model of the human lower extremity fused with a RB model that is validated with experiments and existing qualitative study approaches. Starting with patient bio-data in the form of MRI and/or CT scans of the tissue of interest, a computational model is constructed. This model is then meshed in a preprocessor FE software package or standalone meshing software. The meshed model once refined constitutes the FE model that is then validated for use in non-contact ACL injury studies. The validated FE model includes all major hard and soft tissues of the lower extremity, as well as, the muscles. For model validation in-vitro testing in gait lab is conducted using human subjects instrumented to collect close to injury joint kinematics, muscle activation, and GRFs. These metrics are input to a musculoskeletal RB model which can be implemented via a modeling software. The output from this analysis are the muscle kinematics specifically muscle tendon moment arms and lengths as well as muscle forces. Muscle force data, GRF data, and joint kinematics are input to the FE model and the resulting joint reaction forces and joint moments can be determined and later compared with experiment. Other key output variables from the FE model can include joint contact forces, tibial shear force, tibial displacement relative to femur and ligament forces. An AI tool can be used here to vary the inputs to the FE model until the output metrics are within an order of magnitude of metrics measured in experiment. If the calculated error is within a certain threshold the model can be considered validated. The model can also be modified, constrained, and loaded similarly to other FE modeling, in-vitro, and in-vivo studies in the literature and results compared to aid in model validation. As well, hard and soft tissues material property data in the literature determined using uni-axial or three point bending experiments, can aid in model validation by replicating these experiments in a virtual setting using the tissues of the FE model and comparing the results.

From Fig. (2) it can be gleaned that many disciplines, much hardware and software, many experts, and a high level of effort is required to execute this proposed coupled approach. Nonetheless, this approach allows for virtual experimentation which has significant implication for cost reduction through reduced equipment needs, number of subjects required for testing, and also time for testing. In addition, one of the central aims of this proposed approach is to provide an enabling tool to better capture the many variables, constraints, unknowns, uncertainty, and variability entailed in the complex problem of predicting injury mechanisms and identifying risk factors of non-contact ACL injury. This approach should also be able to simultaneously capture the interaction of multiple forces, risk factors, and other parameters that may contribute to non-contact ACL injury in a seamless automated manner. This approach should also aim to provide information that can connect the cause and effect relationships between ACL loading, injury mechanisms, and risk factors of non-contact ACL injury.

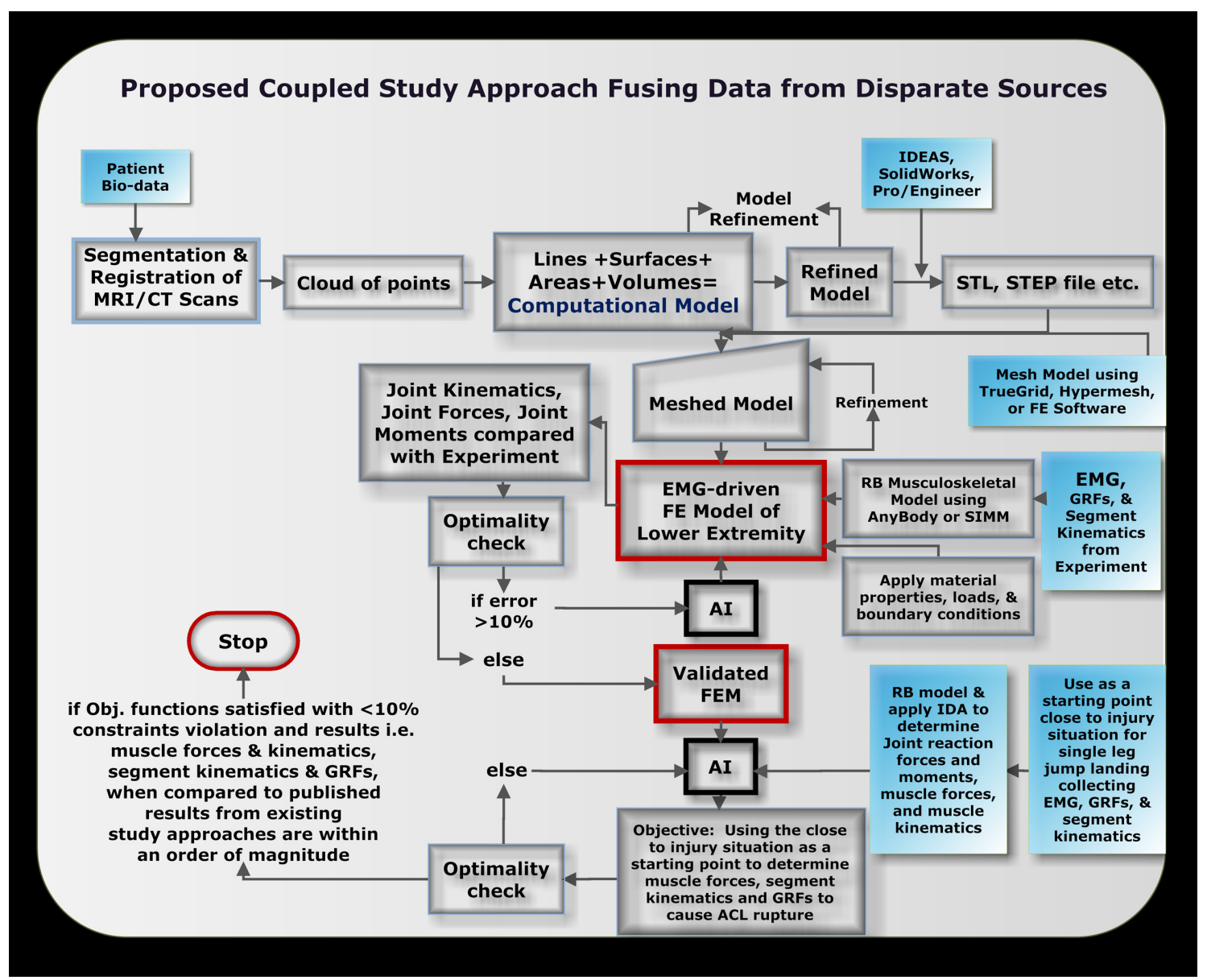

Fig. (2). Possible coupled study approach to better understand mechanisms and risk factors to non-contact ACL injury. 
There are no known quantitative body kinematic data or muscle data that precisely address position of the body at ACL injury during any injury situation. As a result, it soon becomes clear that a standalone detailed validated 3D EMGdriven FE model of the lower extremity may not be able to determine injury mechanisms and identify risk factors of non-contact ACL injury. Hence, determining the lower extremity kinematics at the time of ACL injury using computational models can be challenging. One approach to resolve this challenge may be to conduct parametric and sensitivity studies to isolate risk factors, forces, and other parameters that do not contribute to an ACL injury mechanism. This will then probably reduce the number of contributing risk factors and further allow for the elimination of others. However, apart from having no guarantee that this can be done, this approach is prone to inaccuracies. Isolating one parameter and checking for its effects on ACL strain fails to account for the influence that the isolated parameter has on other surrounding tissue, which may subsequently affect ACL loads. As a result, this approach is not preferred. Due to immense variability in data, as well as the many parameters, constraints, and unknowns incurred in a single multifaceted problem of non-contact ACL injury, a tool is needed to address such a requirement. Moreover, the numerous risk factors which are simultaneously at play during a non-contact ACL injury event, as well as, the high dimensionality, great interdependencies, and temporal dependencies demands the capabilities of some form of an optimization routine. Present non-contact ACL injury study approaches lack the capabilities required to overcome such challenges. It should be noted that classical optimization techniques such as the Newton Raphson search method cannot be applied to the problem of how and why noncontact ACL injuries occur because of the absence of a polynomial type function, and more importantly, it cannot handle many design parameters in a large domain. As an alternative, the authors believe that a stochastic or artificial intelligent (AI) technique should be employed for this purpose. The application of an AI technique to ACL injury biomechanics is relatively new, but provides an avenue to tackle problems involving many parameters, many constraints, and multiple objective functions over a large search domain. An AI technique can also be employed to orchestrate the fusion of various study approaches in a single environment as well as to facilitate search and parameter identification. Mathematical programming and the Monte Carlo method [132, 147] are the current often used optimization approaches employed to study ACL mechanics. Although both mathematical programming and Monte Carlo methods have demonstrated their usefulness and effectiveness as a research tool, there are much more advanced and robust AI techniques; namely, taboo search, simulated annealing, genetic algorithms, and artificial neural networks. For small search spaces, classical exhausted methods usually suffice; however, for larger search spaces special AI techniques must be employed.

Hence, the ability to fuse a validated EMG-driven FE model with an AI technique that allows for automatic or semi-automatic data exchange becomes imperative. This can be achieved through multidisciplinary design optimization (MDO). The author's view is that combining the five existing study approaches using an AI technique in a MDO paradigm maybe a much more robust and comprehensive methodology for predicting non-contact ACL injury mechanisms and risk factors. Multidisciplinary design optimization (MDO) has recently emerged as a field of research and practice that brings together many previously disjointed disciplines and tools. Typically MDO involves many design variables, many constraints, and analysis from various contributing disciplines, where coupling between disciplines and conflicting requirements exist. This proposed MDO paradigm entails the fusion of data from patient, clinical studies, video analysis, athletic interviews, experiment, and computational modeling via an artificial intelligent (AI) technique.

A schematic outline of how this can be accomplished is shown in Fig. (2). To elucidate the validated FE model can be used to study specific non-contact ACL injury mechanisms such as deceleration when landing on one leg from a jump. The inputs to this model are measured in gait lab and muscle kinematics determined from musculoskeletal $\mathrm{RB}$ modeling. An AI tool is then utilized to define a problem to determine the instance where many risk factors, many forces, and other extreme conditions happen simultaneously to cause ACL rupture. To ensure practicality, clinical studies, interviews with athletes' studies, and video analysis studies are consulted during simulation when studying the specific ACL injury causing event. Fig. (2) highlights the key processes entailed in the proposed multidisciplinary approach. The authors believe that exploiting the strengths of various existing non-contact ACL injury study approaches in a combinatory manner may overshadow, yield improved information, and even negate the disadvantages encountered when each approach is employed on its own.

\section{CONCLUSIONS}

This review is geared towards highlighting the barriers to obtaining a better understanding of the injury mechanisms and risk factors to non-contact ACL injury. The ultimate goal of acquiring such an understanding is to improve prevention and training strategies, as well as, improve clinical diagnosis and treatment of ACL injuries. The precise mechanism of injury and combination of risk factors that endanger the ACL are not completely understood, partly because studies do not simultaneously include the effects of the muscles, accurate 3D tissue geometries, as well as, the hip, knee, and ankle articulations on ACL loading. In addition, there are many limitations with experimentation, clinical studies, and other existing study approaches when employed on their own that prohibits a comprehensive understanding of the injury mechanisms and risk factors of non-contact ACL injury. Moreover, the narrow focus of some studies and the dearth of standards and specifications in the field of biomechanics appear to have the effect of limiting progress in reinforcing our understanding of noncontact ACL injury. Given the many barriers to understanding non-contact ACL injury mechanisms and risk factors, it is presently not known which aspect of a prevention program is the key element in preventing ACL injuries, or how they work. A possible approach based on fusing existing study approaches using an AI technique in a MDO environment is proposed. The uniqueness of the proposed study is that it simultaneously captures the interaction of multiple forces and myriad of factors 
implicated as causes of non-contact ACL injury. As well the proposed approach is also capable of handling numerous variables, constraints, and objective functions over a large multidimensional search space as well as great interdependencies, high dimensionality, and temporal dependencies.

Based on this review, it is evident that in spite of significant amount of research related to non-contact ACL injury, there are still many open questions about injury mechanisms and conflicting views as to which factor contributes to risk of injury. There is a great need to develop new research methods capable of addressing the myriad of factors, high dimensionality, and great interdependencies involved in non-contact ACL injury.

\section{AUTHORS' CONTRIBUTION}

NA was responsible for conception, writing, and revising of the manuscript, while GR was responsible for reviewing, drafting, and final approval of the manuscript.

\section{REFERENCES}

[1] M. L. Ireland, M. Gaudette, and S. Crook, "ACL injuries in the female athlete," J. Sport Rehabil., vol. 6, pp. 97-110, 1997.

[2] B. P. Boden, G. S. Dean, A. J. Feagin, and W. E. J. Garrett, "Mechanisms of anterior cruciate ligament injury," Orthopedics, vol. 23, pp. 573-578, 2000.

[3] B. P. Boden, J. S. Torg, S. B. Knowles, and T. E. Hewett, "Video Analysis of Anterior Cruciate Ligament Injury," Am. J. Sports Med., vol. 37, pp. 252-259, 2009.

[4] O.-E. Olsen, G. Myklebust, L. Engebretsen, and R. Bahr, "Injury Mechanisms for Anterior Cruciate Ligament Injuries in Team Handball," Am. J. Sports Med., vol. 32, pp. 1002-1012, 2004.

[5] Y. Nagano, H. Ida, M. Akai, and T. Fukubayashi, "Biomechanical characteristics of the knee joint in female athletes during tasks associated with anterior cruciate ligament injury," The Knee, vol. 16, pp. 153-158, 2009.

[6] M. L. Ireland, "The female ACL: why is it more prone to injury?," Orthop. Clin. N. Am., vol. 33, pp. 637-651, 2002.

[7] P. Renstrom et al., "Non-contact ACL injuries in female athletes: an International Olympic Committee current concepts statement," Br. J. Sports Med., vol. 42, pp. 394-412, 2008.

[8] L. J. Huston, M. L. V. H. Greenfield, and E. M. Wojtys, "Anterior Cruciate Ligament Injuries in the Female Athlete: Potential Risk Factors," Clin. Orthop. Relat. Res., vol. 372, pp. 50-63, 2000.

[9] C. A. M. D. Gottlob, C. L. J. M. D. Baker, J. M. P. Pellissier, and L. P. Colvin, "Cost Effectiveness of Anterior Cruciate Ligament Reconstruction in Young Adults," Clin. Orthop. Relat. Res., vol. 367, pp. 272-282, 1999.

[10] D. M. Daniel and D. Fristschy, Anterior cruciate ligament injuries. vol. 2. Philadelphia: W.B. Saunders, 1994.

[11] C. F. Braden, L. C. James, P. S. Kurt, and M. M. Martha, "Can suture repair of ACL transection restore normal anteroposterior laxity of the knee? An ex vivo study," J. Orthop. Res. , vol. 26, pp. 1500-1505, 2008.

[12] M. E. Eastlack, M. J. Axe, and L. Snyder-Mackler, "Laxity, instability, and functional outcome after ACL injury: copers versus noncopers," Med. Sci. Sports Exercise, vol. 31, pp. 210-215, 1999.

[13] D. Roberts, E. Ageberg, G. Andersson, and T. Fridén, "Clinical measurements of proprioception, muscle strength and laxity in relation to function in the ACL-injured knee," Knee Surg. Sport Tr. A., vol. 15 , pp. 9-16, 2007.

[14] L. Y. Griffin et al., "Noncontact Anterior Cruciate Ligament Injuries: Risk Factors and Prevention Strategies," J. Am. Acad. Orthop. Surg., vol. 8, pp. 141-150, 2000.

[15] W. C. Whiting and R. F. Zernicke, Biomechanics of musculoskeletal injury. Champaign, IL: Human Kinetics, 1998.

[16] E. Ageberg, "Neuromuscular training optimizes knee function after arthroscopic ACL reconstruction.," Aust. J. Physiother., vol. 53, p. 287, 2007.

[17] G. Myer, K. Ford, J. Brent, and T. Hewett, "Differential neuromuscular training effects on ACL injury risk factors in "high- risk" versus "low-risk" athletes," BMC Musculoskeletal Disorders, vol. 8, pp. 39-46, 2007.

[18] A. L. Bryant, J. Kelly, and E. Hohmann, "Neuromuscular adaptations and correlates of knee functionality following ACL reconstruction," J. Orthop. Res., vol. 26, pp. 126-135, 2008.

[19] J. Hashemi, N. Chandrashekar, T. Jang, F. Karpat, M. Oseto, and S. Ekwaro-Osire, "An Alternative Mechanism of Non-contact Anterior Cruciate Ligament Injury During Jump-landing: In-vitro Simulation," Exp. Mech., vol. 47, pp. 347-354, 2007.

[20] J. A. Feagin Jr and K. L. Lambert, "Mechanism of injury and pathology of anterior cruciate ligament injuries," Orthop. Clin. N. Am. , vol. 16, pp. 41-45, 1985.

[21] P. J. McNair, R. N. Marshall, and J. A. Matheson, "Important features associated with acute anterior cruciate ligament injury," $N$ Z Med. J., vol. 103, pp. 537-539, 1990.

[22] C. C. Teitz, "Video analysis of ACL injuries," In Prevention of non-contact ACL injuries (Edited by Griffin,L.Y.), pp. 87-92, 2001.

[23] A. Ferretti, P. Papandrea, F. Conteduca, and P. P. Mariani, "Knee ligament injuries in volleyball players," Am. J. Sports Med., vol. 20, pp. 203-207, 1992.

[24] O. E. Olsen, G. Myklebust, L. Engebretsen, I. Holme, and R. Bahr, "Relationship between floor type and risk of ACL injury in team handball," Scand. J. Med. Sci. Sports, vol. 13, pp. 299-304, 2003.

[25] P. FaunÃ, and B. Wulff Jakobsen, "Mechanism of Anterior Cruciate Ligament Injuries in Soccer," Int. J. Sports Med., vol. 27, pp. 75-79, 2006.

[26] G. Myklebust, S. Maehlum, L. Engebretsen, T. Strand, and E. Solheim, "Registration of cruciate ligament injuries in Norwegian top level team handball. A prospective study covering two seasons," Scand. J. Med. Sci. Sports., vol. 7, pp. 289-292, 1997.

[27] R. Bahr and T. Krosshaug, "Understanding injury mechanisms: a key component of preventing injuries in sport," Br. J. Sports Med., vol. 39, pp. 324-329, 2005.

[28] C. E. Quatman and T. E. Hewett, "The anterior cruciate ligament injury controversy: is "valgus collapse" a sex-specific mechanism?," Br. J. Sports Med., vol. 43, pp. 328-335, 2009.

[29] M. L. Ireland, "Anterior Cruciate Ligament Injury in Female Athletes: Epidemiology," J. Athl. Train., vol. 34, pp. 150-154, 1999.

[30] T. E. Hewett, G. D. Myer, and K. R. Ford, "Anterior Cruciate Ligament Injuries in Female Athletes: Part 1, Mechanisms and Risk Factors," Am. J. Sports Med., vol. 34, pp. 299-311, 2006.

[31] S. G. McLean, K. Walker, K. R. Ford, G. D. Myer, T. E. Hewett, and A. J. van den Bogert, "Evaluation of a two dimensional analysis method as a screening and evaluation tool for anterior cruciate ligament injury," Br. J. Sports Med., vol. 39, pp. 355-362, 2005.

[32] S. G. McLean, "The ACL Injury Enigma: We Can't Prevent What We Don't Understand," J. Athl. Train., vol. 43, pp. 538-540, 2008.

[33] W. H. Meeuwisse, "Assessing Causation in Sport Injury: A Multifactorial Model," Clin. J. Sport Med., vol. 4, pp. 166-170, 1994.

[34] S. J. Shultz, "ACL Injury in the Female Athlete: A Multifactorial Problem That Remains Poorly Understood," J. Athl. Train., vol. 43, p. 455, 2008.

[35] C. E. Quatman, C. C. Quatman, and T. E. Hewett, "Prediction and prevention of musculoskeletal injury: a paradigm shift in methodology," Br. J. Sports Med., vol. 43, pp. 1100-1107, 2009.

[36] D. G. Lloyd, T. F. Besier, C. R. Winby, and T. S. Buchanan, Neuromusculoskeletal modelling and simulation of tissue load in the lower extremities: Routledge, 2008.

[37] M. Viceconti, F. Taddei, L. Montanari, D. Testi, A. Leardini, G. Clapworthy, and S. Van Sint Jan, "Multimod Data Manager: A tool for data fusion," Comput. Meth. Prog. Bio., vol. 87, pp. 148-159, 2007.

[38] J. Ziegler and M. G. Pandy, "A computational model for determining muscle-ligament interactions at the knee during movement," Comput. Med., vol. 23, pp. 532-568, 1995.

[39] S. M. Lephart, J. P. Abt, and C. M. Ferris, "Neuromuscular contributions to anterior cruciate ligament injuries in females," Curr. Opin. Rheumatol., vol. 14, pp. 168-173, 2002.

[40] L. Y. Griffin et al., "Understanding and Preventing Noncontact Anterior Cruciate Ligament Injuries: A Review of the Hunt Valley II Meeting," Am. J. Sports Med., vol. 34, pp. 1512-1532, 2006.

[41] E. A. Arendt, "Musculoskeletal injuries of the knee: are females at greater risk?," Minnesota Med., vol. 90, pp. 38-40, 2007. 
[42] Y. Nagano, H. Ida, M. Akai, and T. Fukubayashi, "Gender differences in knee kinematics and muscle activity during single limb drop landing," The Knee, vol. 14, pp. 218-223, 2007.

[43] B. D. Beynnon, and B. C. Fleming, "Anterior cruciate ligament strain in-vivo: A review of previous work," J. Biomech., vol. 31, pp. 519-525, 1998.

[44] J. P. McConkey, "Anterior cruciate ligament rupture in skiing: A new mechanism of injury," Am. J. Sports Med., vol. 14, pp. 160164, 1986.

[45] K. L. Markolf, G. O'Neill, S. R. Jackson, and D. R. McAllister, "Effects of Applied Quadriceps and Hamstrings Muscle Loads on Forces in the Anterior and Posterior Cruciate Ligaments," Am. J. Sports Med., vol. 32, pp. 1144-1149, 2004.

[46] G. Li, T. W. Rudy, M. Sakane, A. Kanamori, C. B. Ma, and S. L. Y. Woo, "The importance of quadriceps and hamstring muscle loading on knee kinematics and in-situ forces in the ACL," $J$. Biomech., vol. 32, pp. 395-400, 1999.

[47] B. D. Beynnon, B. C. Fleming, R. J. Johnson, C. E. Nichols, P. A. Renstrom, and M. H. Pope, "Anterior Cruciate Ligament Strain Behavior During Rehabilitation Exercises In Vivo," Am. J. Sports Med., vol. 23, pp. 24-34, 1995.

[48] A. K. Aune, P. W. Cawley, and A. Ekeland, "Quadriceps Muscle Contraction Protects the Anterior Cruciate Ligament During Anterior Tibial Translation," Am. J. Sports Med., vol. 25, pp. 187190, 1997.

[49] G. DeMorat, P. Weinhold, T. Blackburn, S. Chudik, and W. Garrett, "Aggressive Quadriceps Loading Can Induce Noncontact Anterior Cruciate Ligament Injury," Am. J. Sports Med., vol. 32, pp. 477-483, 2004.

[50] D. L. Isaac, D. J. Beard, A. J. Price, J. Rees, D. W. Murray, and C. A. F. Dodd, "In-vivo sagittal plane knee kinematics: ACL intact, deficient and reconstructed knees," The Knee, vol. 12, pp. 25-31, 2005.

[51] M. Solomonow, R. Baratta, and R. D'Ambrosia, "The role of the hamstrings in the rehabilitation of the anterior cruciate ligamentdeficient knee in athletes," Sports Med., vol. 7, pp. 42-8, 1989.

[52] K. B. Shelburne and M. G. Pandy, "Determinants of cruciateligament loading during rehabilitation exercise," Clin. Biomech. , vol. 13, pp. 403-413, 1998.

[53] E. B. Simonsen, S. P. Magnusson, J. Bencke, H. Naesborg, M. Havkrog, J. F. Ebstrup, and H. Sorensen, "Can the hamstring muscles protect the anterior cruciate ligament during a side-cutting maneuver?," Scand. J. Med. Sci. Sports, vol. 10, pp. 78-84, 2000.

[54] B. C. Fleming et al., "The gastrocnemius muscle is an antagonist of the anterior cruciate ligament," J. Orthop. Res., vol. 19, pp. 11781184, 2001.

[55] M. A. Pflum, K. B. Shelburne, M. R. Torry, M. J. Decker, and M. G. Pandy, "Model Prediction of Anterior Cruciate Ligament Force during Drop-Landings," Med. Sci. Sports Exercise, vol. 36, pp. 1949-1958, 2004.

[56] L. Durselen, L. Claes, and H. Kiefer, "The Influence of Muscle Forces and External Loads on Cruciate Ligament Strain," Am. J. Sports Med., vol. 23, pp. 129-136, 1995.

[57] K. B. Shelburne and M. G. Pandy, "A musculoskeletal model of the knee for evaluating ligament forces during isometric contractions," J. Biomech., vol. 30, pp. 163-176, 1997.

[58] R. B. Lambson, B. S. Barnhill, and R. W. Higgins, "Football Cleat Design and Its Effect on Anterior Cruciate Ligament Injuries: A Three-Year Prospective Study," Am. J. Sports Med., vol. 24, pp. 155-159, 1996.

[59] A. M. Chaudhari and T. P. Andriacchi, "The mechanical consequences of dynamic frontal plane limb alignment for noncontact ACL injury," J. Biomech., vol. 39, pp. 330-338, 2006.

[60] L. C. Kramer, C. R. Denegar, W. E. Buckley, and J. Hertel, "Factors associated with anterior cruciate ligament injury: history in female athletes," J. Sports Med. Phys. Fit., vol. 47, pp. 446-54, 2007.

[61] F. C. Anderson and M. G. Pandy, "Individual muscle contributions to support in normal walking," Gait Posture, vol. 17, pp. 159-169, 2003.

[62] J. Smith, J. E. Szczerba, B. L. Arnold, D. H. Perrin, and D. E. Martin, "Role of Hyperpronation as a Possible Risk Factor for Anterior Cruciate Ligament Injuries," J. Athl. Train., vol. 32, pp. 25-28, 1997.
[63] P. Devita and W. A. Skelly, "Effect of landing stiffness on joint kinetics and energetics in the lower extremity," Med. Sci. Sports Exerc. , vol. 24, pp. 108-115, 1992.

[64] M. J. Decker, M. R. Torry, D. J. Wyland, W. I. Sterett, and J. Richard Steadman, "Gender differences in lower extremity kinematics, kinetics and energy absorption during landing," Clin. Biomech., vol. 18, pp. 662-669, 2003.

[65] B. Yu, C.-F. Lin, and W. E. Garrett, "Lower extremity biomechanics during the landing of a stop-jump task," Clin. Biomech., vol. 21, pp. 297-305, 2006.

[66] S. G. McLean, X. Huang, and A. J. van den Bogert, "Association between lower extremity posture at contact and peak knee valgus moment during sidestepping: Implications for ACL injury," Clin. Biomech., vol. 20, pp. 863-870, 2005.

[67] J. R. Meakin, N. G. Shrive, C. B. Frank, and D. A. Hart, "Finite element analysis of the meniscus: the influence of geometry and material properties on its behaviour," The Knee, vol. 10, pp. 33-41, 2003.

[68] J. Zeiss, K. Paley, K. Murray, and S. R. Saddemi, "Comparison of Bone Contusion Seen by MRI in Partial and Complete Tears of the Anterior Cruciate Ligament," J. Comput. Assist Tomogr., vol. 19, pp. 773-776, 1995.

[69] G. Li, T. W. Rudy, C. Allen, M. Sakane, and S. L. Woo, "Effect of combined axial compressive and anterior tibial loads on in situ forces in the anterior cruciate ligament: A porcine study," $J$. Orthop. Res., vol. 16, pp. 122-127, 1998.

[70] W. Liu and M. E. Maitland, "Influence of Anthropometric and Mechanical Variations on Functional Instability in the ACLDeficient Knee," Ann. Biomed. Eng., vol. 31, pp. 1153-1161, 2003.

[71] P. Renstrom, S. W. Arms, T. S. Stanwyck, R. J. Johnson, and M. H. Pope, "Strain within the anterior cruciate ligament during hamstring and quadriceps activity," Am. J. Sports Med., vol. 14, pp. 83-87, 1986.

[72] M. Z. Bendjaballah, A. Shirazi-Adl, and D. J. Zukor, "Biomechanics of the human knee joint in compression: reconstruction, mesh generation and finite element analysis," The Knee, vol. 2, pp. 69-79, 1995.

[73] C. Bratianu, P. Rinderu, and L. Gruionu, "A 3D finite element model of a knee for joint contact stress analysis during sport activities," Key Engineering Materials Vols., vol. 261, pp. 513-518, 2004.

[74] C.-F. Lin, M. Gross, C. Ji, D. Padua, P. Weinhold, W. E. Garrett, and $\mathrm{B}$. Yu, "A stochastic biomechanical model for risk and risk factors of non-contact anterior cruciate ligament injuries," $J$. Biomech., vol. 42, pp. 418-423, 2009.

[75] T. Krosshaug, T. E. Andersen, O. E. O. Olsen, G. Myklebust, and R. Bahr, "Research approaches to describe the mechanisms of injuries in sport: limitations and possibilities," Br. J. Sports Med., vol. 39, pp. 330-339, 2005.

[76] B. C. Fleming, P. A. Renstrom, B. D. Beynnon, B. Engstrom, G. D. Peura, G. J. Badger, and R. J. Johnson, "The effect of weightbearing and external loading on anterior cruciate ligament strain," J. Biomech., vol. 34, pp. 163-170, 2001.

[77] S. L. Y. Woo, S. D. Abramowitch, R. Kilger, and R. Liang, "Biomechanics of knee ligaments: injury, healing, and repair," $J$. Biomech., vol. 39, pp. 1-20, 2006.

[78] R. B. Frobell, H. P. Roos, E. M. Roos, M. P. Hellio Le Graverand, R. Buck, J. Tamez-Pena, S. Totterman, T. Boegard, and L. S. Lohmander, "The acutely ACL injured knee assessed by MRI: are large volume traumatic bone marrow lesions a sign of severe compression injury?," Osteoarthr. Cartilage, vol. 16, pp. 829-836, 2008.

[79] G. Li, T. H. Wuerz, and L. E. DeFrate, "Feasibility of Using Orthogonal Fluoroscopic Images to Measure In Vivo Joint Kinematics," J. Biomech. Eng., vol. 126, pp. 313-318, 2004.

[80] G. Li, L. E. DeFrate, S. E. Park, T. J. Gill, and H. E. Rubash, "In Vivo Articular Cartilage Contact Kinematics of the Knee," Am. J. Sports Med., vol. 33, pp. 102-107, 2005.

[81] R. Stagni, S. Fantozzi, A. Cappello, and A. Leardini, "Quantification of soft tissue artefact in motion analysis by combining 3D fluoroscopy and stereophotogrammetry: a study on two subjects," Clin. Biomech., vol. 20, pp. 320-329, 2005.

[82] G. Li, L. E. DeFrate, H. E. Rubash, and T. Gill, "In vivo kinematics of the ACL during weight-bearing knee flexion," J. Orthop. Res., vol. 23, pp. 340-344, 2005. 
[83] S. L. Woo, M. A. Gomez, Y. K. Woo, and W. H. Akeson, "Mechanical properties of tendons and ligaments. II. The relationships of immobilization and exercise on tissue remodeling," Biorheology, vol. 19, pp. 397-408, 1982.

[84] R. van Dijk, R. Huiskes, and G. Selvik, "Roentgen stereophotogrammetric methods for the evaluation of the three dimensional kinematic behaviour and cruciate ligament length patterns of the human knee joint," J. Biomech., vol. 12, pp. 727$731,1979$.

[85] R. C. M. B. Meijer, R. Huiskes, and J. M. G. Kauer, "A stereophotogrammetric method for measurements of ligament structure," J. Biomech., vol. 22, pp. 177-179, 1989.

[86] C. E. Henning, M. A. Lynch, and K. R. J. R. Glick, "An in vivo strain gage study of elongation of the anterior cruciate ligament," Am. J. Sports Med., vol. 13, pp. 22-26, 1985.

[87] J. P. Holden, E. S. Grood, D. L. Korvick, J. F. Cummings, D. L. Butler, and D. I. Bylski-Austrow, "In vivo forces in the anterior cruciate ligament: Direct measurements during walking and trotting in a quadruped," J. Biomech., vol. 27, pp. 517-519, 1994.

[88] B. C. Fleming et al., "Determination of a zero strain reference for the anteromedial band of the anterior cruciate ligament," J. Orthop. Res., vol. 12 pp. 789-795, 1994.

[89] B. D. Beynnon et al., "The measurement of elongation of anterior cruciate-ligament grafts in vivo," J. Bone Joint Surg., vol. 76, pp. 520-531, 1994.

[90] K. L. Markolf, J. F. Gorek, J. M. Kabo, and M. S. Shapiro, "Direct measurement of resultant forces in the anterior cruciate ligament. An in vitro study performed with a new experimental technique," $J$. Bone Joint Surg., vol. 72, pp. 557-567, 1990.

[91] G. Cerulli, D. L. Benoit, M. Lamontagne, A. Caraffa, and A. Liti, "In vivo anterior cruciate ligament strain behaviour during a rapid deceleration movement: case report," Knee Surg. Sport Tr. A., vol. 11, pp. 307-311, 2003.

[92] D. Barry and A. M. Ahmed, "Design and performance of a modified buckle transducer for measurement of ligament tension," J. Biomech. Eng., vol. 108, pp. 149-152, 1986.

[93] A. M. Ahmed, D. L. Burke, N. A. Duncan, and K. H. Chan, "Ligament tension pattern in the flexed knee in combined passive anterior translation and axial rotation," J. Orthop. Res., vol. 8, pp. 854 - 867, 1992.

[94] A. M. Ahmed, A. Hyder, D. L. Burke, and K. H. Chan, "In-vitro ligament tension pattern in the flexed knee in passive loading," $J$. Orthop. Res., vol. 5, pp. 217-230, 1987.

[95] J. Lewis, Lew WD, Hill JA, Ohland KJ, Kirkstukus S, Hunter RE, "Knee joint motion and ligament forces before and after ACL recontruction," J. Biomech. Eng., vol. 111, pp. 97-106, 1989.

[96] K. N. An, Berflund, L, Cooney, W P, Chao E Y S, Kovacevic N., "Direct in-vivo tendon force measurement system," J. Biomech. Eng., vol. 23, pp. 1269-1271, 1990.

[97] P. V. Komi, "Relevance of in vivo force measurements to human biomechanics," J. Biomech., vol. 23, pp. 23-25, 1990.

[98] T. J. Withrow, L. J. Huston, E. M. Wojtys, and J. A. Ashton-Miller, "The Relationship between Quadriceps Muscle Force, Knee Flexion, and Anterior Cruciate Ligament Strain in an In Vitro Simulated Jump Landing," Am. J. Sports Med., vol. 34, pp. 269274, 2006.

[99] T. J. A. Mommersteeg, R. Huiskes, L. Blankevoort, J. G. M. Kooloos, and J. M. G. Kauer, "An inverse dynamics modeling approach to determine the restraining function of human knee ligament bundles," J. Biomech., vol. 30, pp. 139-146, 1997.

[100] J. L. Haimes, R. R. Wroble, E. S. Grood, and F. R. Noyes, "Role of the Medial Structures in the intact and Anterior Cruciate LigamentDeficient Knee: Limits of Motion in the Human Knee," Am. J. Sports Med., vol. 22, pp. 402-409, 1994.

[101] K. L. Markolf, D. M. Burchfield, M. M. Shapiro, M. F. Shepard, G. A. M. Finerman, and J. L. Slauterbeck, "Combined knee loading states that generate high anterior cruciate ligament forces," $J$. Orthop. Res., vol. 13, pp. 930-935, 1995.

[102] D. L. Benoit, D. K. Ramsey, M. Lamontagne, L. Xu, P. Wretenberg, and P. Renstrom, "Effect of skin movement artifact on knee kinematics during gait and cutting motions measured in vivo," Gait Posture, vol. 24, pp. 152-164, 2006.

[103] S. Tashman, D. Collon, K. Anderson, P. Kolowich, and W. Anderst, "Abnormal Rotational Knee Motion During Running After Anterior Cruciate Ligament Reconstruction," Am. J. Sports Med., vol. 32, pp. 975-983, 2004.
[104] C. Reinschmidt, A. J. van den Bogert, B. M. Nigg, A. Lundberg, and N. Murphy, "Effect of skin movement on the analysis of skeletal knee joint motion during running," J. Biomech., vol. 30, pp. 729-732, 1997.

[105] R. Crowninshield, M. H. Pope, and R. J. Johnson, "An analytical model of the knee," J. Biomech., vol. 9, pp. 397-405, 1976.

[106] M. S. Hefzy and T. D. V. Cooke, "Review of Knee Models: 1996 Update," Appl. Mech. Rev., vol. 49, pp. 187-193, 1996.

[107] W. A. M. Brekelmans, H. W. Poort, and T. Slooff, "A New Method to Analyse the Mechanical Behaviour of Skeletal Parts," Acta Orthop. Scand., vol. 43, pp. 301-317, 1972.

[108] R. Huiskes and S. J. Hollister, "From Structure to Process, From Organ to Cell: Recent Developments of FE-Analysis in Orthopaedic Biomechanics," J. Biomech. Eng., vol. 115, pp. 520527, 1993.

[109] D. Brown Thomas, "Finite Element Modeling in Musculoskeletal Biomechanics," J. Appl. Biomech. , vol. 20, pp. 336-366, 2004.

[110] T. P. Andriacchi, R. P. Mikosz, S. J. Hampton, and J. O. Galante, "Model studies of the stiffness characteristics of the human knee joint," J. Biomech., vol. 16, pp. 23-29, 1983.

[111] E. Peña, B. Calvo, M. A. Martínez, D. Palanca, and M. Doblaré, "Finite element analysis of the effect of meniscal tears and meniscectomies on human knee biomechanics," Clin. Biomech., vol. 20, pp. 498-507, 2005.

[112] Y. Song, R. E. Debski, V. Musahl, M. Thomas, and S. L. Y. Woo, "A three-dimensional finite element model of the human anterior cruciate ligament: a computational analysis with experimental validation," J. Biomech., vol. 37, pp. 383-390, 2004.

[113] A. G. Au, V. J. Raso, A. B. Liggins, D. D. Otto, and A. Amirfazli, "A three-dimensional finite element stress analysis for tunnel placement and buttons in anterior cruciate ligament reconstructions," J. Biomech., vol. 38, pp. 827-832, 2005.

[114] K. E. Moglo and A. Shirazi-Adl, "Cruciate coupling and screwhome mechanism in passive knee joint during extension-flexion," J. Biomech., vol. 38, pp. 1075-1083, 2005.

[115] N. A. Ramaniraka, A. Terrier, N. Theumann, and O. Siegrist, "Effects of the posterior cruciate ligament reconstruction on the biomechanics of the knee joint: a finite element analysis," Clin. Biomech., vol. 20, pp. 434-442, 2005.

[116] E. Peña, B. Calvo, M. A. Martínez, D. Palanca, and M. Doblaré, "Computational Modelling of Diarthrodial Joints. Physiological, Pathological and Pos-Surgery Simulations," Arch. Comput. Methods Eng., vol. 14, pp. 47-91, 2007.

[117] N. A. Ramaniraka, P. Saunier, O. Siegrist, and D. P. Pioletti, "Biomechanical evaluation of intra-articular and extra-articular procedures in anterior cruciate ligament reconstruction: A finite element analysis," Clin. Biomech., vol. 22, pp. 336-343, 2007.

[118] T. Sawatari, H. Tsumura, K. Iesaka, Y. Furushiro, and T. Torisu, "Three-dimensional finite element analysis of unicompartmental knee arthroplasty - the influence of tibial component inclination," J. Orthop. Res., vol. 23, pp. 549-554, 2005.

[119] J. J. Elias and A. J. Cosgarea, "Computational Modeling: An Alternative Approach for Investigating Patellofemoral Mechanics," Sports Med. Arthrosc., vol. 15, pp. 89-94, 2007.

[120] P. Beillas, G. Papaioannou, S. Tashman, and K. H. Yang, "A new method to investigate in vivo knee behavior using a finite element model of the lower limb," J. Biomech., vol. 37, pp. 1019-1030, 2004.

[121] J. Zhiheng, D. Zhijiang, and W. Monan, "A Novel Finite Element Method based Biomechanical Model for HIT-Robot Assisted Orthopedic Surgery System," in 28th Annual International Conference of the IEEE, 2006, pp. 1735-1738.

[122] C. Untaroiu, K. Darvish, J. Crandall, B. Deng, and J. T. Wang, "Characterization of the lower limb soft tissues in pedestrian finite element models," in 19th International technical conference on the Enhanced Safety of Vehicles, Washington DC, 2005, pp. 124-131.

[123] A. Jilani, A. Shirazi-Adl, and M. Z. Bendjaballah, "Biomechanics of human tibio-femoral joint in axial rotation," The Knee, vol. 4, pp. 203-213, 1997.

[124] T. L. H. Donahue and M. L. Hull, "A Finite Element Model of the Human Knee Joint for the Study of Tibio-Femoral Contact," $J$. Biomech. Eng., vol. 124, pp. 273-280, 2002.

[125] G. Li, J. Gil, A. Kanamori, and S. L. Y. Woo, "A Validated ThreeDimensional Computational Model of a Human Knee Joint," $J$. Biomech. Eng., vol. 121, pp. 657-662, 1999. 
[126] J. M. T. Penrose, G. M. Holt, M. Beaugonin, and D. R. Hose, "Development of An Accurate Three-dimensional Finite Element Knee Model," Comput. Meth. Biomech. Biomed. Eng., vol. 5, pp. 291-300, 2002.

[127] D. Périé and M. C. Hobatho, "In vivo determination of contact areas and pressure of the femorotibial joint using non-linear finite element analysis," Clin. Biomech., vol. 13, pp. 394-402, 1998.

[128] M. Z. Bendjaballah, A. Shirazi-Adl, and D. J. Zukor, "Finite element analysis of human knee joint in varus-valgus," Clin. Biomech., vol. 12, pp. 139-148, 1997.

[129] G. Limbert, M. Taylor, and J. Middleton, "Three-dimensional finite element modelling of the human ACL: simulation of passive knee flexion with a stressed and stress-free ACL," J. Biomech., vol. 37, pp. 1723-1731, 2004.

[130] M. Gabriel, E. Wong, S. Woo, M. Yagi, and R. Debski, "Distribution of in situ forces in the anterior cruciate ligament in response to rotatory loads," J. Orthop. Res., vol. 22, pp. 85-89, 2004.

[131] B. J. Fregly, Y. Bei, and M. E. Sylvester, "Experimental evaluation of an elastic foundation model to predict contact pressures in knee replacements," J. Biomech., vol. 36, pp. 1659-1668, 2003.

[132] J. Suggs, C. Wang, and G. Li, "The effect of graft stiffness on knee joint biomechanics after ACL reconstruction-a 3D computational simulation," Clin. Biomech., vol. 18, pp. 35-43, 2003.

[133] M. G. Pandy, K. Sasaki, and S. Kim, "A Three-Dimensional Musculoskeletal Model of the Human Knee Joint. Part 1: Theoretical Construction," Comput. Meth. Biomech. Biomed. Eng., vol. 1, pp. 87-108, 1997.

[134] S. G. McLean, A. Su, and A. J. van den Bogert, "Development and Validation of a 3-D Model to Predict Knee Joint Loading During Dynamic Movement," J. Biomech. Eng., vol. 125, pp. 864-874, 2003.

[135] F. C. Anderson and M. G. Pandy, "Static and dynamic optimization solutions for gait are practically equivalent," J. Biomech., vol. 34, pp. 153-161, 2001.

[136] M. Viceconti, S. Olsen, L. P. Nolte, and K. Burton, "Extracting clinically relevant data from finite element simulations," Clin. Biomech., vol. 20, pp. 451-454, 2005.
[137] C. B. Payne and A. R. Bird, "Teaching clinical biomechanics in the context of uncertainty," J. Am. Podiat. Med. Assoc., vol. 89, pp. 525-530, 1999.

[138] D. F. Murphy, D. A. J. Connolly, and B. D. Beynnon, "Risk factors for lower extremity injury: a review of the literature," Br. J. Sports Med., vol. 37, pp. 13-29, 2003.

[139] K. G. Harmon and M. L. Ireland, "Gender differences in noncontact anterior cruciate ligament injuries " Clin. Sports Med., vol. 19, pp. 287-302, 2000.

[140] M. G. Horton and T. L. Hall, "Quadriceps femoris muscle angle: normal values and relationships with gender and selected skeletal measures," Phys. Ther., vol. 69, pp. 897-901, 1989.

[141] M. D. Tillman, K. R. Smith, J. A. Bauer, J. H. Cauraugh, A. B. Falsetti, and J. L. Pattishall, "Differences in three intercondylar notch geometry indices between males and females: a cadaver study," The Knee, vol. 9, pp. 41-46, 2002.

[142] E. M. Wojtys, L. J. Huston, T. N. Lindenfeld, T. E. Hewett, and M. Greenfield, "Association Between the Menstrual Cycle and Anterior Cruciate Ligament Injuries in Female Athletes," Am. J. Sports Med., vol. 26, pp. 614-619, 1998.

[143] R. A. Malinzak, S. M. Colby, D. T. Kirkendall, B. Yu, and W. E. Garrett, "A comparison of knee joint motion patterns between men and women in selected athletic tasks," Clin. Biomech., vol. 16, pp. 438-445, 2001.

[144] NIH, "The National Library of Medicine's Visible Human Project," http://www.nlm.nih.gov/research/visible/visible_human.html., 2000.

[145] S. V. S. Jan, "The VAKHUM project: virtual animation of the kinematics of the human," Theor. Issues Ergonomics Sci., vol. 6, pp. 277-279, 2005.

[146] M. Viceconti, M. Ansaloni, M. Baleani, and A. Toni, "The muscle standardized femur: a step forward in the replication of numerical studies in biomechanics," J. Eng. Med., vol. 217, pp. 105-110, 2003.

[147] L. Blankevoort and R. Huiskes, "Validation of a three-dimensional model of the knee," J. Biomech., vol. 29, pp. 955-961, 1996.

Received: April 16, 2010

Revised: July 23, 2010

Accepted: July 25, 2010

(C) Ali and Rouhi; Licensee Bentham Open.

This is an open access article licensed under the terms of the Creative Commons Attribution Non-Commercial License (http://creativecommons.org/licenses/by-nc/3.0/) which permits unrestricted, non-commercial use, distribution and reproduction in any medium, provided the work is properly cited. 